\section{Schweizerische Akademie der Medizinischen Wissenschaften}

«Prix Excellence»: erstmalige Ausschreibung des SAMW-Medienpreises

Die Schweizerische Akademie der Medizinischen Wissenschaften (SAMW) sieht es als ihre Aufgabe an, den Dialog zwischen der Medizin und der Gesellschaft zu fördern; sie wird dabei unterstützt von Journalisten/Journalistinnen und Forschenden, welche komplexe Themen für ein breites Publikum aufbereiten. Mit dem neu geschaffenen Medienpreis möchte die SAMW diese Vermittlungsarbeit würdigen und fördern. Der «Prix Excellence» in der Höhe von Fr. 10000 .- zeichnet in Zukunft jährlich einen Beitrag von herausragender Qualität aus, der sich zu einem von der SAMW vorgängig bezeichneten Thema an ein Laienpublikum richtet und der in einem täglich oder wöchentlich erscheinenden Schweizer Medium publiziert wird. Jedes Jahr legt der Senat der SAMW ein Preisthema fest. Preisthema 2005: «Die Zukunft der Medizin».

\section{Teilnahmebedingungen}

Verliehen wird der «Prix Excellence» an Journalistinnen und Journalisten oder Forschende, die mit einem journalistischen Beitrag auf hervorragende Weise einem nicht spezialisierten Publikum das Thema «Die $\mathrm{Zu}$ kunft der Medizin» nahebringen, womöglich mit Bezug zum Projekt «Zukunft Medizin Schweiz» der SAMW. In ihrer Beurteilung legt die Jury besonderes Augenmerk auf den gelungenen Wissenstransfer. Berücksichtigt werden Printmedien (Zeitung oder Zeitschrift), die täglich oder wöchentlich in der Schweiz erscheinen, oder Radio- und Fernsehsendungen, die im Tages- oder Wochenrhythmus ausgestrahlt werden. Der eingereichte Beitrag muss zwischen dem 1. Januar 2005 und 31. Dezember 2005 publiziert bzw. ausgestrahlt worden sein. Er wird von einer sieben- köpfigen Jury aus Medien und Wissenschaft beurteilt. Das Reglement des Preises sowie der Bericht «Ziele und Aufgaben der Medizin zu Beginn des 21. Jahrhunderts» können auf der Homepage der Akademie (www.samw.ch $\rightarrow$ Publikationen) eingesehen oder telefonisch angefordert werden.

Preisverleihung: Der Preis wird anlässlich der Sitzung des SAMW-Senats vom 23. Mai 2006 verliehen.

Einsendeschluss: Einsendeschluss für die Kandidaturen ist der 28. Februar 2006 (Datum des Poststempels). Bei audiovisuellen Beiträgen ist den Aufnahmen in einem gängigen Format eine kurze Beschreibung des Inhalts beizulegen.

Die Arbeiten sind an die Adresse der Akademie zu richten: SAMW, Petersplatz 13, 4051 Basel, Tel. 06126990 30, Fax 06126990 39, Kontaktperson: Dr. Hermann Amstad, E-Mail mail@samw.ch, Internet: www.samw.ch.

\section{Académie Suisse des Sciences Médicales}

«Prix Excellence»: première mise au concours du prix médiatique de l'ASSM

L'Académie Suisse des Sciences Médicales (ASSM) considère qu'il est de son devoir de promouvoir le dialogue entre la médecine et la société; elle est soutenue, dans cette tâche, par des journalistes et des chercheurs qui publient des articles sur des sujets complexes en les rendant accessibles à un large public. Avec ce nouveau prix médiatique, l'ASSM aimerait honorer et encourager ce travail d'intermédiaire. Le «Prix Excellence» d'un montant de Fr. 10000 .- sera décerné à l'auteur d'un article de grande qualité consacré à un sujet défini par l'ASSM, destiné à un public profane et paraissant dans un média quotidien ou heb- domadaire suisse. C'est le Sénat de l'ASSM qui, chaque année, détermine le thème; en 2005 le sujet choisi est «L'avenir de la médecine».

\section{Conditions de participation}

Le Prix Excellence sera décerné à des journalistes ou des chercheurs capables, à travers un écrit de grande qualité, de familiariser un public non spécialisé avec le thème de «L'avenir de la médecine», si possible avec référence au projet «L'avenir de la médecine en Suisse» de l'ASSM. Dans son évaluation, le jury prêtera particulièrement attention à la qualité du transfert des connaissances. Il sera tenu compte, d'une part des articles de presse (journaux ou magazines) paraissant en Suisse et, d'autre part, des émissions radiodiffusées ou télévisées, qu'ils soient quotidiens ou hebdomadaires. L'article ou l'émission présenté doit avoir été publié ou diffusé entre le $1^{\mathrm{er}}$ janvier et le 31 décembre 2005. Il sera évalué par un jury composé de sept membres issus des domaines des médias et de la science. Le règlement concernant l'attribution de ce prix ainsi que le rapport «Buts et missions de la médecine au début du $21^{\text {e }}$ siècle» peuvent être consultés sur le site internet de l'Académie (www.samw.ch $\rightarrow$ publications) ou commandés par téléphone.

Remise $d u$ prix: Le prix sera remis dans le cadre de la séance du Sénat de l'ASSM du 23 mai 2006.

Date limite des envois: La date limite des envois des candidatures est le 28 février 2006 (date du cachet de la poste). Lorsqu'il s'agit de documents audiovisuels, un bref descriptif sera joint aux enregistrements en format courant.

Les travaux doivent être adressés à l'Académie: ASSM, Petersplatz 13, 4051 Bâle, tél. 061 26990 30, fax: 06126990 39, Contact: Dr Hermann Amstad, e-mail: mail@samw.ch, internet: www.assm.ch. 